\title{
The influence of fish consumption on serum $n-3$ polyunsaturated fatty acid (PUFA) concentrations in women of childbearing age: a randomised controlled trial (the iFish Study)
}

\author{
Marie C. Conway ${ }^{1} \cdot$ Emeir M. McSorley $^{1} \cdot$ Maria S. Mulhern ${ }^{1} \cdot$ Toni Spence $^{1} \cdot$ Edwin van Wijngaarden $^{2}$. \\ Gene E. Watson ${ }^{2} \cdot$ Karin Wahlberg $^{3} \cdot$ Daniela Pineda $^{3} \cdot$ Karin Broberg $^{3,4} \cdot$ Barry W. Hyland $^{5} \cdot$ Diego F. Cobice ${ }^{5}$. \\ J. J. Strain ${ }^{1} \cdot$ Alison J. Yeates ${ }^{1}[1$
}

Received: 12 February 2020 / Accepted: 1 July 2020 / Published online: 28 July 2020

(c) The Author(s) 2020

\begin{abstract}
Purpose Long-chain polyunsaturated fatty acids (LCPUFA) can be synthesised endogenously from linoleic acid (LA) and $\alpha$-linolenic acid (ALA) in a pathway involving the fatty acid desaturase (FADS) genes. Endogenous synthesis is inefficient; therefore, dietary intake of preformed LCPUFA from their richest source of fish is preferred. This study investigated the effect of fish consumption on PUFA concentrations in women of childbearing age while stratifying by FADS genotype. The influence of fish consumption on lipid profile, and markers of inflammation and oxidative stress was also examined.

Methods Healthy women $(n=49)$ provided a buccal swab which was analysed for FADS2 genotype (rs3834458; T/deletion). Participants were stratified according to genotype and randomised to an intervention group to receive either no fish $(n=18)$, 1 portion $(n=14)$ or 2 portions $(n=17)$ ( $140 \mathrm{~g}$ per portion) of fish per week for a period of 8 weeks. Serum PUFA was analysed at baseline and post-intervention. Lipid profile, and markers of inflammation and oxidative stress were also analysed. Results Participants consuming 2 portions of fish per week had significantly higher concentrations of eicosapentaenoic acid (EPA), docosahexaenoic acid (DHA) and total n-3 PUFA, and a lower n-6:n-3 ratio compared to those in the no fish or 1 portion per week group (all $p<0.05$ ). Fish consumption did not have a significant effect on biomarkers of oxidative stress, inflammation and lipid profile in the current study.

Conclusion Consumption of 2 portions of fish per week has beneficial effects on biological n-3 PUFA concentrations in women of childbearing age; however, no effects on oxidative stress, inflammation or lipid profile were observed. This trial was registered at www.clinicaltrials.gov (NCT03765580), registered December 2018.
\end{abstract}

Keywords Fish consumption · Polyunsaturated fatty acids $\cdot$ Eicosapentaenoic acid $\cdot$ Docosahexaenoic acid $\cdot$ Fatty acid desaturase $\cdot$ FADS

Electronic supplementary material The online version of this article (https://doi.org/10.1007/s00394-020-02326-w) contains supplementary material, which is available to authorized users.

Alison J. Yeates

a.yeates@ulster.ac.uk

1 Nutrition Innovation Centre for Food and Health (NICHE), School of Biomedical Sciences, Ulster University, Cromore Road, Coleraine BT52 1SA, Northern Ireland, UK

2 School of Medicine and Dentistry, University of Rochester, Rochester, USA

3 The Laboratory of Medicine, Division of Occupational and Environmental Medicine, Lund University, Lund, Sweden

\section{Abbreviations \\ AA Arachidonic acid \\ ALA $\alpha$-Linolenic acid \\ ANCOVA Analysis of covariance}

4 Institute of Environmental Medicine, Karolinska Institutet, Stockholm, Sweden

5 Mass Spectrometry Centre, Biomedical Sciences Research Institute (BMSRI), Ulster University, Coleraine, Northern Ireland, UK 


$\begin{array}{ll}\text { BMI } & \text { Body mass index } \\ \text { DHA } & \text { Docosahexaenoic acid } \\ \text { EPA } & \text { Eicosapentaenoic acid } \\ \text { FADS } & \text { Fatty acid desaturase } \\ \text { GCMS } & \text { Gas chromatography mass spectrometry } \\ \text { HISU } & \text { Human intervention studies unit } \\ \text { IL } & \text { Interleukin } \\ \text { LA } & \text { Linoleic acid } \\ \text { LCPUFA } & \text { Long-chain polyunsaturated fatty acids } \\ \text { PUFA } & \text { Polyunsaturated fatty acid } \\ \text { RCT } & \text { Randomised controlled trial } \\ \text { SPSS } & \text { Statistical package for social sciences } \\ \text { UREC } & \text { University research ethics committee }\end{array}$

\section{Introduction}

Polyunsaturated fatty acids (PUFA) have important roles in health, including a role in the inflammatory response, cell membrane structure and function, and are particularly important during pregnancy [1]. Arachidonic acid (AA) and docosahexaenoic acid (DHA) are essential for brain development [2-4], particularly during the third trimester of pregnancy when DHA accumulation in the brain is most rapid $[5,6]$. Higher PUFA status in pregnant women has also been linked to improved child cognitive outcomes [7-12], and has been found to have a role in reducing inflammation, oxidative stress and lipid profile [13-15].

Linoleic acid (LA) and $\alpha$-linolenic acid (ALA) are essential fatty acids which cannot be synthesised in vivo and must be provided by the diet [16]. These precursor molecules go through a series of elongation and desaturation steps to produce long-chain (LC) PUFA including n-6 AA, and eicosapentaenoic acid (EPA) and DHA of the n-3 family. The fatty acid desaturase 1 (FADS1) and FADS2 genes are located on chromosome 11q12-q13.1, and encode the $\Delta-5$ and $\Delta-6$ desaturase enzymes (D5D and D6D respectively) involved in the elongation and desaturation pathway [17]. Single nucleotide polymorphisms (SNPs) in FADS1 and FADS2 have been reported to influence LCPUFA status, with minor allele carriers having lower concentrations of DHA, EPA and AA [18-23]. Even in individuals with high LCPUFA intake, with increasing number of minor alleles of FADS2 rs3834458, the concentrations of AA were significantly decreased [18]. This SNP is in strong linkage disequilibrium [24] with other FADS SNPs, e.g. rs174537 and rs174545, that have also been linked to LCPUFA status [18, 20, 22, 23]. Thus, rs 3834458 tags functional variation of the FADS region. The endogenous synthesis pathway, however, is inefficient [25], and thus, dietary intake of preformed LCPUFA is preferred to meet requirements.
Fish is a rich source of many nutrients including PUFA. Fish consumption in the UK is below the current recommendations of two portions of fish per week [26]. Increased fish consumption is hypothesised to improve biological status of LCPUFA [27]; nevertheless, there are conflicting findings with regard to LCPUFA dietary intake from fish and biological LCPUFA status. Increased fish consumption has been found to be associated with increased PUFA status in pregnant women [28, 29], and non-pregnant, adult populations [30, 31]. Research to date has not conclusively shown an increase in LCPUFA status following increased dietary intake, with some researchers reporting no association between fish consumption and LCPUFA status [9, 32, 33]. Studies have focused on fish consumption of pregnant women [9], and in mothers and their newborns [33, 34] in observational and intervention studies, respectively. The observational study by Bonham et al. [9] may have lacked associations owing to physiological factors of pregnancy, such as the transfer of PUFA from mother to foetus. An observational study in non-pregnant adults concluded associations between fish consumption and biological PUFA status cannot be generalised to all fish intakes, with fatty fish influencing biological LCPUFA status; whereas, the consumption of lean fish does not. The authors suggest these findings may be owing to EPA and DHA being metabolised into plasma differently [32]. It is also important to acknowledge that factors other than diet are associated with PUFA status, such as sex, physical activity and genetics [35]. During pregnancy, the mobilisation of DHA into circulation may alter erythrocyte PUFA profile, and subsequently lead to a lower correlation between circulating PUFA status and dietary intake [29].

The evidence available to date for associations between fish consumption and PUFA status is mainly observational [9, 27-32]. There is a lack of studies intervening with fish consumption to investigate whether consumption will increase biological PUFA status. In an intervention study in pregnant women who were low habitual consumers of fish, women were randomised to consume either 2 portions of salmon per week or to continue their habitual diet [34]. This study concluded that the consumption of 2 portions of salmon per week will increase EPA and DHA biological status in pregnant women who do not regularly consume oily fish. The role of genetics has not been controlled for in studies completed to date, and thus, FADS SNPs may account in part for the discrepancies in the influence of fish consumption on PUFA status. The conversion of the precursors LA and ALA to LCPUFA may be greater in those consuming less fish [36]; therefore, given the influence of the FADS genotype on the endogenous synthesis pathway, it is possible that individuals with the minor allele for FADS may benefit from direct consumption of preformed EPA and DHA. Research in women of childbearing age 
is of importance owing to the key role of PUFA during pregnancy and for child development.

To our knowledge, no randomised controlled trials (RCTs) intervening with fish have considered confounding by FADS genotype. Therefore, the primary aim of the current study was to investigate whether fish consumption influences serum n-3 PUFA concentrations in women of childbearing age while stratifying by FADS genotype. It was hypothesised that fish consumption will increase concentrations of n-3 LCPUFA in a dose-response manner when allowing for a major confounding factor of genetic variation in FADS2 (rs3834458, T/deletion). Fish consumption has been shown to decrease biomarkers of inflammation [37], oxidative stress [38] and lipid profile [39]. The effect of fish intervention on these markers was also assessed in the current study in secondary analysis. The influence of fish consumption on serum n-3 PUFA concentrations within genotype groups for carriers of the rs3834458 (TT) and for carriers of the minor allele (Tdel or deldel) was also investigated in secondary analysis.

\section{Methods}

\section{Study design and population}

The "iFish Study" (registered at www.clinicaltrials.gov (NCT03765580)) was an 8-week RCT with the overall aim of investigating whether fish consumption influences PUFA status in women of childbearing age when accounting for FADS genotype. Female participants were recruited within Ulster University, Coleraine, UK and the surrounding area from October 2016. All those interested in taking part in the study completed a screening questionnaire. Inclusion criteria were: being a healthy female of childbearing age (aged between 18 and 45 years), premenopausal, and not planning on becoming pregnant during the course of the study. Furthermore, suitable participants had to be low consumers of fish $(<2$ portions of fish per week), willing to consume 1,2 or no portions of fish per week, non-consumers of fish oil or protein supplements, and not allergic to seafood. Exclusion criteria included: being a regular consumer of fish and not willing to do washout period where they reduced fish consumption in advance of the study; allergic to seafood, taking fish oil or protein supplements, being pregnant or menopausal, or having very short hair. All participants provided written informed consent and the study was approved by Ulster University Research Ethics Committee (REC/16/0077). All research was conducted in accordance with the 1964 Declaration of Helsinki and its amendments.

\section{SNP selection and genotype determination}

Eligible participants provided a buccal swab. Buccal swab samples were stored at $-80{ }^{\circ} \mathrm{C}$ and shipped to Lund University, Sweden for DNA extractions and genotyping. DNA was isolated using the QIAamp DNA Mini Kit (Qiagen, Hilden, Germany) according to the manufacturer's protocol. Rs3834458 was genotyped on an ABI 7900HT Fast Real Time PCR System (Applied Biosystems, Thermo Fisher, Waltham, Massachusetts, USA) using a custom TaqMan assay (Thermo Fisher Scientific) according to the manufacturer's instructions. As mentioned, rs3834458 was selected based on its strong associations with PUFA status [18, 40]. This SNP is referred to as an intronic variant and has a minor allele frequency (MAF) of 35\% in European populations [24]. As a quality control measure, approximately 5\% of samples were randomly selected and reanalysed. There was $100 \%$ agreement between original and duplicate samples. Further quality assessment of the data was completed by evaluating Hardy-Weinberg equilibrium using Chi-Square test.

\section{Recruitment and randomisation}

Recruitment of participants involved the distribution of information emails, posters and leaflets within Ulster University, Coleraine, UK and the surrounding area. A total of 124 women expressed interest in the study while 89 completed the screening questionnaire, of which 66 were deemed eligible and were recruited. Power calculations were based on observing a difference in PUFA status with an effect size of 1.8 [41] in a within-between ANOVA using G-Power with a power level of $80 \%$ and significance level of 0.05 . It was determined that a total of 10 people were required for each of the three intervention groups (no fish, 1 portion and 2 portions of fish per week). Participants were stratified based on rs3834458 genotype and randomised into an intervention group of either no fish, 1 portion or 2 portions of fish per week by an independent Clinical Trials Manager using MINIM software (MINIM, UK). Of the eligible participants randomised after genotyping, 17 individuals did not commence the study ( $n=7$ did not wish to take part any longer; and $n=10$ were not contactable to arrange a baseline appointment). These 17 individuals were equally distributed across the intervention groups and there were no significant differences in age or fish consumption between those who took part and those who did not commence the study. Fortynine apparently healthy females began the study.

\section{Intervention period}

Participants were invited to attend two sampling appointments at the Human Intervention Study Unit (HISU) at 
Ulster University at baseline and post-intervention beginning in January 2017. At each time point, participants provided biological samples including blood, and information on habitual dietary intake, anthropometric measurements and general health and lifestyle information was also recorded. Study participants were provided with a lunch dish either once or twice a week (depending on their intervention group) in the HISU. Each lunch contained a 140-g portion of fish according to the intervention group to which they were randomised (either tuna or sardines for the duration of the study). Those randomised to the no fish group also received a lunchtime meal with no fish once per week. Participants were given the option of choosing between a salad, baked potato or sandwich lunch. Each lunchtime option was entered on Nutritics dietary analysis software (Nutritics Ltd, Swords, Dublin) to calculate energy and macronutrient composition to ensure similar amounts were provided across each of the intervention groups. Leftovers were weighed and recorded to measure compliance. The same batch numbers were used throughout the study for the tinned tuna and sardines, and samples of the fish were analysed for PUFA concentrations (ALS Life Sciences, UK) prior to the study commencing. Total $\mathrm{n}-3$ concentrations $(\mathrm{g} / 100 \mathrm{~g})$ were 4.57 for tuna, and 6.47 for sardines.

\section{Blood sampling and anthropometric measurements}

Fasting blood samples collected at baseline and post-intervention were processed to obtain serum and plasma by centrifugation at $3500 \mathrm{rpm}$ for $15 \mathrm{~min}$ at $4{ }^{\circ} \mathrm{C}$. An aliquot of plasma had $0.005 \%$ butylated hydroxytoluene (BHT) added as an antioxidant at the time of blood processing. All aliquots were subsequently frozen and stored at $-80{ }^{\circ} \mathrm{C}$ until batch analysis. Body weight (to the nearest $0.1 \mathrm{~kg}$ ) was measured using TANITA digital scales (TANITA Europe, The Netherlands). Height was measured to the nearest centimetre using a stadiometer and body mass index (BMI, kg/ $\mathrm{m}^{2}$ ) was then calculated.

\section{PUFA analysis}

Serum samples were analysed for total PUFA, using a method adapted from Folch et al. [42]. This method involved extraction of total lipids followed by methylation to fatty acid methyl esters (FAME) using boron trifluoride methanol $\left(\mathrm{BF}_{3}\right)$ (Sigma Aldrich, UK) [43]. FAME were quantified using gas chromatography mass spectrometry (GC-MS) (7890A-5975C; Agilent Technologies UK Ltd, UK). Analysis was completed in split mode, with a BPX70 capillary GC column (SGE Analytical Science) (length $30 \mathrm{~m}$, internal diameter $250 \mu \mathrm{m}$ and film thickness $0.25 \mu \mathrm{m}$ ), using helium as the carrier gas (constant flow at $1.0 \mathrm{ml} / \mathrm{min}$; purity 99.9999\%). Samples were injected using an automatic liquid sampler (ALS) (injection volume $1 \mu \mathrm{l}$ ) at a temperature of $130{ }^{\circ} \mathrm{C}$; which was then ramped at $15{ }^{\circ} \mathrm{C} / \mathrm{min}$ to $200^{\circ} \mathrm{C}$ and then at $30{ }^{\circ} \mathrm{C} / \mathrm{min}$ to $250{ }^{\circ} \mathrm{C}$ where it was held for $5 \mathrm{~min}$. Mass spectrometry was operated in positive ion mode using an electron ionisation (EI) source. Mass range was set to 50-500 Da and acquisition was performed by total ion chromatogram (TIC). LA, ALA, AA, EPA and DHA were identified by their retention time and corresponding qualifier ions with reference to those of commercially available fatty acid standards (Sigma Aldrich, UK), and were quantified by use of an internal standard, heptadecanoic acid (C17:0) (Sigma Aldrich, UK) and corresponding PUFA target ions (quantifiers). For the current study, total $\mathrm{n}-6(\mathrm{mg} / \mathrm{ml})$ was calculated by summing LA and AA concentrations, and ALA, EPA and DHA were summed to calculate total $\mathrm{n}-3(\mathrm{mg} / \mathrm{ml})$. The n-6:n-3 ratio was also calculated.

\section{Biomarker analyses}

Biomarkers known to be influenced by n-3 PUFA were analysed. Serum lipids were analysed using the I-LAB 650 Chemical Analyser (Instrumentation Laboratories, Warrington, Cheshire, UK). LDL cholesterol was calculated using the Friedewald formula (Total cholesterol - HDL - (triglycerides/2.2)). The I-LAB 650 Chemical Analyser was also used to measure high-sensitivity $\mathrm{C}$-reactive protein (hsCRP) in serum samples. The inflammatory markers interleukin (IL)-5, IL-10, IL-1 $\beta$, IL-6 and tumour necrosis factor-alpha (TNF- $\alpha$ ) were measured using an immunoassay from Meso Scale Discovery (MSD, Meso Scale Diagnostics, LLC, Maryland, USA). Samples with concentrations of inflammatory markers below the lower limit of detection (LLOD) had the LLOD/ $\sqrt{ } 2$ inputted. Samples of whole blood were analysed for glutathione peroxidase (GPx), a marker of oxidative stress, using the Ransal Assay (Randox, UK) on the I-LAB 650 Chemical Analyser. At the time of processing, an aliquot of plasma had $0.005 \%$ BHT to allow for analysis of 8-isoprostanes. The plasma samples were then purified using 8-isoprostane affinity sorbent (Cayman Chemical, Ann Arbor, MI, USA), and analysed using an 8-isoprostane ELISA kit supplied by Cayman Chemical.

\section{Dietary intake and general health}

Participants completed a 24-h recall of all foods and beverages consumed in the previous $24 \mathrm{~h}$ at both baseline and post-intervention appointments. Dietary intakes were quantified using Nutritics software (Nutritics Ltd, 2018). Participants also completed a health and lifestyle questionnaire to provide information on general health, including supplement usage, personal medical history and smoking and alcohol drinking habits, as well as demographic and socioeconomic data. At each lunchtime meal, participants were asked if they 
had eaten any fish in addition to the study portion. If additional fish was eaten, details including the type and quantity eaten were obtained from the participant.

\section{Statistical analysis}

Statistical analyses of the data were completed using SPSS (Statistical Package for Social Sciences, Version 24.0. SPSS UK Ltd., Chertsey, UK). All statistical analyses were completed per protocol, and also using intention to treat (ITT). For ITT analyses, baseline values of subjects lost to follow-up were entered as post-intervention values [44]. All data were tested for normality, and skewed data were log transformed to approximate normality. Descriptive analyses were performed, and all data are expressed as median and interquartile range (25th, 75 th percentiles). Differences in baseline characteristics between the three intervention groups were analysed using one-way analysis of variance (ANOVA).

In the primary analysis, analysis of covariance (ANCOVA), with LSD for post hoc comparison controlling for age, BMI and baseline serum PUFA concentrations, was used to investigate the effect of fish intervention on serum PUFA concentrations in the cohort stratified by genotype. Furthermore, ANCOVA analyses, controlling for age, BMI and relevant baseline concentrations were conducted to assess the effect of intervention on biomarkers of oxidative stress, inflammation and on lipid profile. In secondary analyses, participants within each treatment group were assigned into one of two genotype groups and the ANCOVA was repeated for homozygous carriers of the rs 3834458 major allele (TT, $n=21$ ) and for carriers of the minor allele (Tdel or deldel, $n=28$ ) to assess the effect of intervention within genotype.

\section{Results}

The CONSORT flow diagram outlines the number of people screened and recruited on to the study, and those who subsequently completed the intervention (Fig. 1). A total of 66 eligible participants for which buccal swabs were provided were randomised to 3 intervention groups. Of the 66 participants randomised, 49 completed a baseline appointment and began intervention. Three participants ( 1 from each intervention group) were dropouts from the study and were, therefore, absent at a follow-up post-intervention appointment (Fig. 1). Overall compliance was high (98\%) as determined using the weight of lunchtime meals consumed. Compliance for each intervention group was $99 \%$, 98\% and $97 \%$ for no fish, 1 portion, and 2 portions groups, respectively. The median (IQR) age for this cohort was 23 (20, 30 years), with all participants being low consumers of fish ( $<2$ portions per week) at baseline. There were no significant differences in baseline characteristics between intervention groups (Table 1). FADS2 rs3834458 was in Hardy-Weinberg equilibrium $\left(\chi^{2} p\right.$ value $\left.=0.66\right)$. Genotype distribution among the intervention groups for rs 3834458 is shown in Table 1.

Results from ITT and per protocol analyses did not differ and ITT analysis is reported. The effect of intervention on serum PUFA concentrations at week 8 , adjusting for covariates (baseline PUFA concentration, baseline age, and baseline BMI), is shown in Table 2. Intervention with 2 portions of fish per week significantly increased concentrations of EPA, DHA and total n-3 PUFA compared to consumption of no fish or one portion per week (all $p<0.05$ ). The n-6:n-3 ratio was significantly lower for those in the two portions per week group compared to those in the no fish $(\mathrm{p}=0.002)$ and one portion $(p=0.012)$ groups. There was no significant difference in serum PUFA concentrations at post-intervention between those consuming no fish and those consuming 1 portion per week. There were no significant differences in n-6 PUFA (LA, AA, total n-6) concentrations between intervention groups consuming different amounts of fish. No significant effects of intervention were found on concentrations of biomarkers of oxidative stress, inflammation or lipid profile analysed in the current study (Table 3). Baseline values for these biomarkers are shown in Online Resource 1. Post-intervention dietary intake data indicate that there was no significant difference in dietary intake of n-3 PUFA between intervention groups (Online Resource 2), or between genotype groups (Online Resource 3).

Following secondary analyses, two-way ANCOVA showed no interaction with genotype when investigating effects of intervention on PUFA. The effect of intervention within genotype was investigated (Table 4). Homozygous carriers of the major allele (TT), who consumed two portions of fish per week had significantly higher concentrations of EPA ( $p=0.013)$, DHA $(p=0.001)$ and total $n-3$ PUFA ( $p=0.014)$ compared to those in the no fish group. Post-intervention AA concentrations were significantly higher in the no fish group compared to the 1 portion of fish group $(p=0.016)$. The major allele homozygous genotype (TT) showed no significant effect of intervention on the $n-6: n-3$ ratio. Carriers of the minor allele (Tdel or deldel), who consumed two portions of fish per week had significantly higher total $n$-3 PUFA concentrations $(p=0.013)$ compared to those in the no fish group, but in contrast to the TT carriers, there was no significant increase in EPA or DHA concentrations. Furthermore, the minor allele carriers showed no significant effect of intervention on $n-6$ PUFA concentrations, or on the $n$ 6: $n-3$ ratio. Comparison of post-intervention serum PUFA concentrations between homozygous carriers of the major allele (TT) and carriers of the minor allele (Tdel, deldel) 


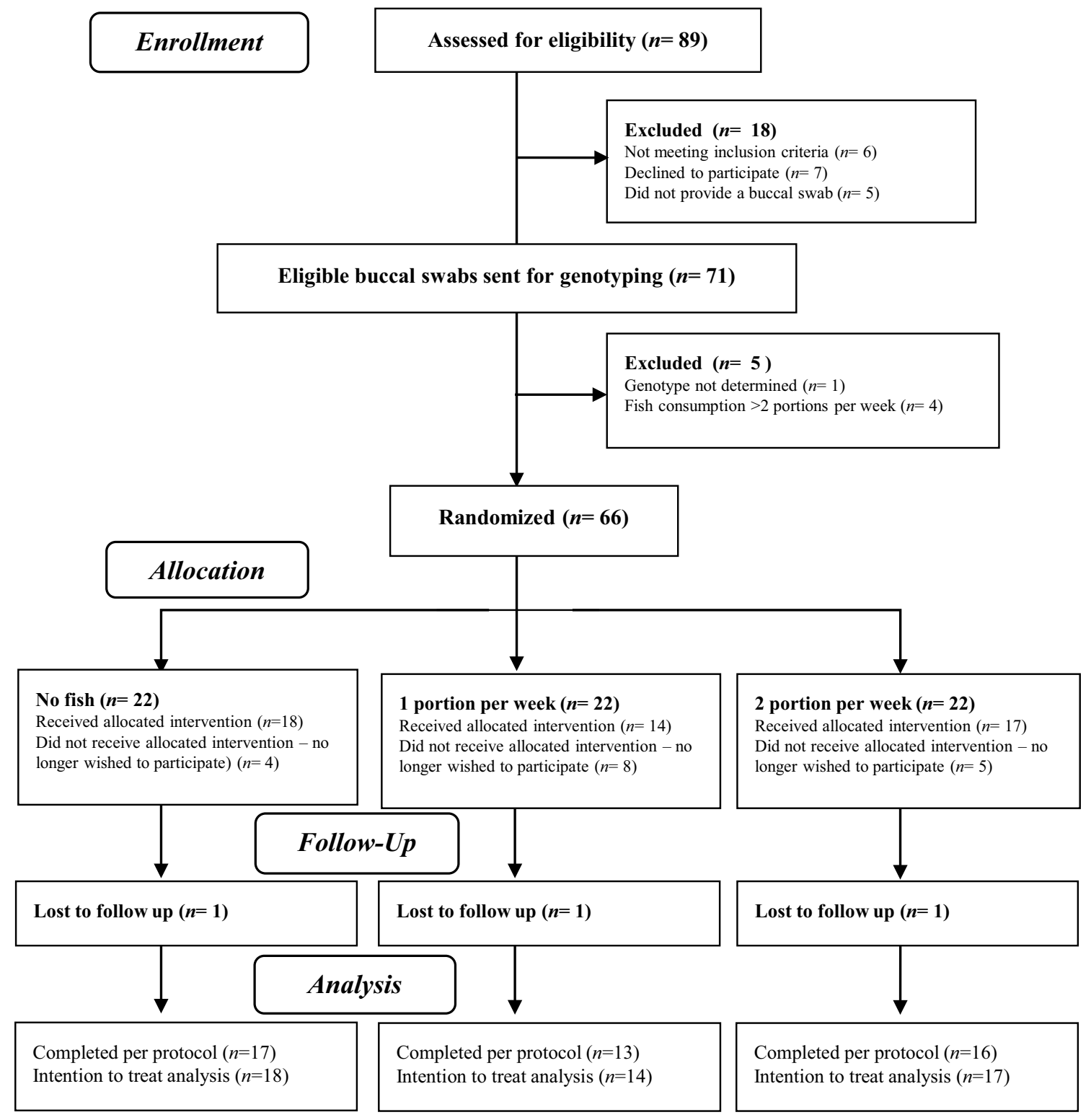

Fig. 1 CONSORT flow diagram for study design

is shown in Online Resource 4. Those who were carriers of the minor allele (Tdel, deldel) had significantly lower serum AA concentrations $(p=0.027)$ and a lower AA:LA ratio $(p=0.001)$ compared to major allele homozygotes (TT). No differences between homozygous carriers of the major allele (TT) and carriers of the minor allele (Tdel, deldel) were seen for any other PUFA. When comparison of post-intervention serum PUFA concentrations between homozygous carriers of the major allele (TT) and carriers of the minor allele (Tdel, deldel) was examined according to intervention group, higher ratios of AA:LA $(p=0.042)$ and DHA:ALA $(p=0.030)$ were found in major allele homozygotes (TT) for those consuming 2 portions of fish per week (Online Resource 5).

\section{Discussion}

To our knowledge, this is the first RCT which investigated the effects of increasing fish consumption on serum PUFA concentrations taking account of FADS genotype. Findings from this study indicate that consumption of two portions of fish per week significantly increases serum total PUFA concentrations of EPA, DHA and total $n-3$ compared to those consuming no fish or 1 portion per week when allowing for a major confounding factor of genetic variation in FADS2 (rs3834458, T/deletion). There was also a significant decrease in the $n-6: n-3$ ratio for those in the 2 portion group compared to the other intervention groups. 
Table 1 Characteristics of iFish study participants at baseline for the whole cohort, and according to intervention group

\begin{tabular}{|c|c|c|c|c|c|}
\hline & Whole group $(n=49)$ & No fish $(n=18)$ & 1 portion $(n=14)$ & 2 portions $(n=17)$ & $P$-value* \\
\hline Age (years) & $23.0(20.0,30.0)$ & $25.5(21.0,33.0)$ & $23.0(19.0,31.8)$ & $22.0(20.0,25.0)$ & 0.766 \\
\hline Weight (kg) & $62.7(57.1,74.3)$ & $68.0(55.9,75.2)$ & $61.0(55.3,69.4)$ & $64.2(57.3,74.1)$ & 0.513 \\
\hline Height (m) & $1.67(1.63,1.70)$ & $1.64(1.62,1.71)$ & $1.67(1.62,1.69)$ & $1.67(1.64,1.71)$ & 0.676 \\
\hline BMI (kg/m2) & $22.7(20.7,26.4)$ & $23.5(21.4,26.9)$ & $21.9(20.3,24.3)$ & $23.3(20.2,25.5)$ & 0.394 \\
\hline Fish consumption (portions/wk) & $1.00(0.50,1.00)$ & $0.75(0.00,1.00)$ & $1.00(0.50,1.25)$ & $1.00(0.50,1.00)$ & 0.516 \\
\hline \multicolumn{6}{|l|}{ Consumes alcohol } \\
\hline Yes & $41(83.7)$ & $17(94.4)$ & $10(71.4)$ & $14(82.4)$ & \multirow[t]{2}{*}{0.214} \\
\hline No & $8(16.3)$ & $1(5.6)$ & $4(28.6)$ & $3(17.6)$ & \\
\hline \multicolumn{6}{|l|}{ Smoker } \\
\hline Yes & $5.0(10.2)$ & $2(11.1)$ & $3(21.4)$ & $0(0)$ & \multirow[t]{2}{*}{0.144} \\
\hline No & $44(89.8)$ & $16(88.9)$ & $11(78.6)$ & $17(100)$ & \\
\hline \multicolumn{6}{|l|}{ Education } \\
\hline Secondary education & $18(36.7)$ & $7(38.9)$ & $5(35.7)$ & $6(35.3)$ & \\
\hline FE college & $5(10.2)$ & $0(0.0)$ & $3(21.4)$ & $2(11.8)$ & \\
\hline Undergraduate (BSc) & $11(22.4)$ & $3(16.7)$ & $4(28.6)$ & $4(23.5)$ & \multirow[t]{3}{*}{0.257} \\
\hline Postgraduate (MSc, PhD) & $12(24.5)$ & $6(33.3)$ & $1(7.1)$ & $5(29.4)$ & \\
\hline Other & $2(4.1)$ & $2(11.1)$ & $0(0.0)$ & $0(0.0)$ & \\
\hline \multicolumn{6}{|l|}{ Employment } \\
\hline Work part time & $11(22.4)$ & $1(5.6)$ & $5(35.7)$ & $5(29.4)$ & \multirow{3}{*}{0.141} \\
\hline Work full time & $8(16.3)$ & $2(11.1)$ & $2(14.3)$ & $4(23.5)$ & \\
\hline Student & $30(61.2)$ & $15(83.3)$ & $7(50.0)$ & $8(27.1)$ & \\
\hline \multicolumn{6}{|l|}{ Genotype (n) } \\
\hline $\mathrm{TT}$ & $21(42.9)$ & $8(44.4)$ & $7(50.0)$ & $6(35.3)$ & \multirow{3}{*}{0.884} \\
\hline $\mathrm{T} / \mathrm{del}$ & $22(44.9)$ & $8(44.4)$ & $6(42.9)$ & $8(47.1)$ & \\
\hline del & $6(12.2)$ & $2(11.1)$ & $1(7.1)$ & $3(17.6)$ & \\
\hline \multicolumn{6}{|l|}{ Baseline PUFA concentrations } \\
\hline $\mathrm{LA}(\mathrm{mg} / \mathrm{ml})$ & $0.263(0.233,0.304)$ & $0.247(0.212,0.305)$ & $0.258(0.224,0.310)$ & $0.273(0.253,0.301)$ & 0.559 \\
\hline $\operatorname{ALA}(\mathrm{mg} / \mathrm{ml})$ & $0.012(0.012,0.014)$ & $0.012(0.011,0.013)$ & $0.012(0.012,0.013)$ & $0.013(0.012,0.014)$ & 0.142 \\
\hline AA (mg/ml) & $0.066(0.054,0.077)$ & $0.067(0.052,0.075)$ & $0.062(0.052,0.076)$ & $0.070(0.056,0.082)$ & 0.778 \\
\hline EPA (mg/ml) & $0.012(0.011,0.014)$ & $0.012(0.011,0.015)$ & $0.012(0.010,0.013)$ & $0.013(0.011,0.014)$ & 0.108 \\
\hline DHA (mg/ml) & $0.021(0.018,0.025)$ & $0.024(0.018,0.026)$ & $0.020(0.017,0.024)$ & $0.020(0.018,0.026)$ & 0.485 \\
\hline Total n-6 (mg/ml) & $0.324(0.295,0.375)$ & $0.318(0.271,0.366)$ & $0.312(0.286,0.374)$ & $0.343(0.310,0.375)$ & 0.563 \\
\hline Total n-3 (mg/ml) & $0.045(0.041,0.052)$ & $0.049(0.041,0.053)$ & $0.042(0.040,0.048)$ & $0.046(0.042,0.053)$ & 0.232 \\
\hline n-6:n-3 ratio & $6.979(6.457,7.810)$ & $6.579(5.688,7.279)$ & $7.342(6.554,8.181)$ & $7.174(6.558,7.916)$ & 0.126 \\
\hline AA:LA ratio & $0.255(0.217,0.295)$ & $0.262(0.216,0.307)$ & $0.257(0.216,0.284)$ & $0.247(0.216,0.292)$ & 0.843 \\
\hline EPA:ALA ratio & $0.990(0.909,1.098)$ & $0.991(0.930,1.145)$ & $0.974(0.839,1.012)$ & $0.994(0.906,1.114)$ & 0.257 \\
\hline DHA:ALA ratio & $1.696(1.456,2.044)$ & $1.778(1.575,2.115)$ & $1.553(1.445,1.895)$ & $1.696(1.340,2.049)$ & 0.335 \\
\hline
\end{tabular}

Data are median (IQR), where IQR is the 25 th and 75 th centiles; or $\mathrm{n}(\%)$ where appropriate; BMI body mass index, FE college Further education college, $L A$ linoleic acid, $A L A \alpha$-linolenic acid, $A A$ arachidonic acid, EPA eicosapentaenoic acid, DHA docosahexaenoic acid

* $p$-value for significant difference between intervention groups at baseline as determined using ANOVA, or Chi square as appropriate; $p<0.05$ considered significant

The current UK and USA dietary recommendations are to consume between two to three portions of fish per week, with at least one of these being oily fish $[45,46]$. This study supports these recommendations as two portions of fish per week significantly increased $n$-3 LCPUFA status over 8 weeks in those who are habitually low fish consumers. Our findings confirm those of Miles et al. [34] who reported an increase in LCPUFA status in pregnant women consuming 2 portions of fish per week who were not habitual consumers of oily fish. Previous studies investigating the influence of fish consumption on PUFA status have not consistently found positive associations between fish intake and PUFA concentrations. Some studies have concluded that fish intake is not associated with $n$-3 PUFA status in pregnant women 
Table 2 The effect of dietary intervention with fish on post-intervention serum PUFA concentrations

\begin{tabular}{|c|c|c|c|c|c|}
\hline PUFA (mg/ml) & No fish $(n=18)$ & 1 portion $(n=14)$ & 2 portions $(n=17)$ & $P$-value & $\begin{array}{l}\text { Partial Eta } \\
\text { squared }\end{array}$ \\
\hline LA & $0.272(0.232,0.300)$ & $0.261(0.246,0.322)$ & $0.294(0.258,0.322)$ & 0.377 & 0.044 \\
\hline ALA & $0.013(0.012,0.014)$ & $0.012(0.012,0.013)$ & $0.013(0.012,0.015)$ & 0.375 & 0.045 \\
\hline AA & $0.065(0.055,0.075)$ & $0.070(0.058,0.086)$ & $0.069(0.059,0.079)$ & 0.375 & 0.045 \\
\hline EPA & $0.012(0.011,0.013)^{\mathrm{a}}$ & $0.014(0.011,0.015)^{\mathrm{a}}$ & $0.014(0.013,0.025)^{\mathrm{b}}$ & $<0.001$ & 0.311 \\
\hline DHA & $0.021(0.016,0.024)^{\mathrm{a}}$ & $0.022(0.020,0.029)^{\mathrm{a}}$ & $0.028(0.024,0.034)^{\mathrm{b}}$ & $<0.001$ & 0.347 \\
\hline Total $n-6$ & $0.343(0.295,0.364)$ & $0.329(0.317,0.406)$ & $0.375(0.318,0.392)$ & 0.396 & 0.042 \\
\hline Total $n-3$ & $0.045(0.042,0.052)^{\mathrm{a}}$ & $0.046(0.038,0.054)^{\mathrm{a}}$ & $0.058(0.051,0.068)^{\mathrm{b}}$ & $<0.001$ & 0.348 \\
\hline$n 6: n 3$ ratio & $7.284(6.586,8.122)^{\mathrm{a}}$ & $7.388(6.992,7.963)^{\mathrm{a}}$ & $6.199(4.897,7.071)^{b}$ & 0.003 & 0.234 \\
\hline AA:LA ratio & $0.243(0.217,0.297)$ & $0.241(0.218,0.278)$ & $0.234(0.214,0.276)$ & 0.411 & 0.040 \\
\hline EPA:ALA ratio & $0.933(0.846,1.058)$ & $1.056(0.889,1.201)$ & $1.043(0.981,1.993)$ & 0.416 & 0.040 \\
\hline DHA:ALA ratio & $1.590(1.345,1.934)$ & $1.823(1.599,2.451)$ & $2.168(1.689,2.603)$ & 0.345 & 0.048 \\
\hline
\end{tabular}

Data are median (IQR), where IQR is the 25th and 75th centiles; PUFA: polyunsaturated fatty acids; LA: linoleic acid; ALA: $\alpha$-linolenic acid; AA: arachidonic acid; EPA: eicosapentaenoic acid; DHA docosahexaenoic acid; total $n-6$ : LA + AA; total $n-3$ : ALA + EPA + DHA; ANCOVA for effect of intervention, adjusting for baseline PUFA, baseline age, baseline BMI; $p<0.05$ considered significant; different superscript letters represent significant differences between groups (LSD post hoc tests); logged variables for those not normally distributed

Table 3 The effect of dietary intervention with fish on biomarker status at post-intervention

\begin{tabular}{|c|c|c|c|c|c|}
\hline Biomarker & No fish $(n=18)$ & 1 portion $(n=14)$ & 2 portions $(n=17)$ & $P$-value & $\begin{array}{l}\text { Partial Eta } \\
\text { squared }\end{array}$ \\
\hline \multicolumn{6}{|c|}{ Lipids ( $\mathrm{mmol} / \mathrm{L})$} \\
\hline Trigs & $0.753(0.590,0.890)$ & $0.695(0.478,1.075)$ & $0.625(0.445,0.828)$ & 0.403 & 0.041 \\
\hline Total chol & $4.300(3.500,4.625)$ & $3.925(3.500,4.675)$ & $4.200(3.725,4.500)$ & 0.865 & 0.007 \\
\hline HDL & $1.485(1.353,1.655)$ & $1.405(1.340,1.470)$ & $1.530(1.335,1.720)$ & 0.902 & 0.005 \\
\hline LDL & $2.300(1.809,2.688)$ & $2.018(1.896,2.818)$ & $2.147(1.903,2.751)$ & 0.607 & 0.023 \\
\hline TC:HDL & $2.632(2.393,2.943)$ & $2.744(2.518,3.463)$ & $2.685(2.299,2.971)$ & 0.881 & 0.006 \\
\hline non-HDL & $2.625(2.205,2.975)$ & $2.440(2.165,3.325)$ & $2.510(2.140,3.020)$ & 0.777 & 0.012 \\
\hline \multicolumn{6}{|c|}{ Inflammatory markers $(\mathrm{pg} / \mathrm{ml})$} \\
\hline IL-5 & $4.074(1.362,11.164)$ & $1.773(1.027,4.278)$ & $2.412(1.149,5.125)$ & 0.165 & 0.080 \\
\hline IL-10 & $0.312(0.181,0.636)$ & $0.373(0.153,0.518)$ & $0.506(0.245,0.700)$ & 0.388 & 0.043 \\
\hline IL- $1 \beta$ & $0.120(0.071,0.183)$ & $0.101(0.029,0.157)$ & $0.161(0.125,0.295)$ & 0.641 & 0.020 \\
\hline IL-6 & $0.627(0.337,1.391)$ & $0.619(0.377,0.805)$ & $0.490(0.365,0.814)$ & 0.679 & 0.018 \\
\hline TNF- $\alpha$ & $2.634(2.385,3.391)$ & $2.873(1.488,3.785)$ & $2.933(2.346,3.624)$ & 0.983 & 0.001 \\
\hline $\mathrm{CRP}(\mu \mathrm{g} / \mathrm{dL})$ & $127.500(39.250,392.750)$ & $68.500(32.000,219.250)$ & $75.000(32.500,281.500)$ & 0.899 & 0.005 \\
\hline \multicolumn{6}{|c|}{ Oxidative stress } \\
\hline GPx (U/L) & $9563.250(7907.875,10,398.625)$ & $8620.250(6959.750,10,439.625)$ & $9655.500(7697.705,11,326.250)$ & 0.343 & 0.048 \\
\hline 8-iso (pg/nl) & $44.666(35.527,65.207)$ & $29.412(22.026,70.417)$ & $37.762(30.384,49.739)$ & 0.335 & 0.050 \\
\hline
\end{tabular}

Data expressed as median (IQR), where IQR is the 25th and 75th centiles; Trigs: Triglycerides; Total chol: Total cholesterol; HDL: High Density Lipoprotein; LDL: Low Density Lipoprotein; TC: Total cholesterol; IL: Interleukin; TNF- $\alpha$ : Tumour necrosis factor-alpha; CRP: C-reactive protein; GPx: Glutathione peroxidase; 8-iso: 8-isoprostanes; ANCOVA: Analysis of covariance; ANCOVA for effect of intervention, adjusting for baseline age, BMI, baseline biomarker status); P-value $\leq 0.05$ considered significant; different letters represent significant differences from each other from LSD post hoc; logged variables for those not normally distributed

[9, 33] and non-pregnant populations [32]. In studies of pregnant women, the lack of an association was suggested to be owing to the increased transfer of $n$-3 LCPUFA to the developing foetus in the third trimester [9, 33]. In an observational study in non-pregnant adults, it was suggested that the metabolism of EPA and DHA from different fish oils may vary with fatty fish influencing biological LCPUFA status, but consumption of lean fish not having an influence on status. Also, a high dietary intake of $n$-6 LCPUFA may result in a decrease in $n$-3 LCPUFA absorption owing to competition for enzymes [32]. None of these studies have taken account of FADS genotype, which may have influenced 


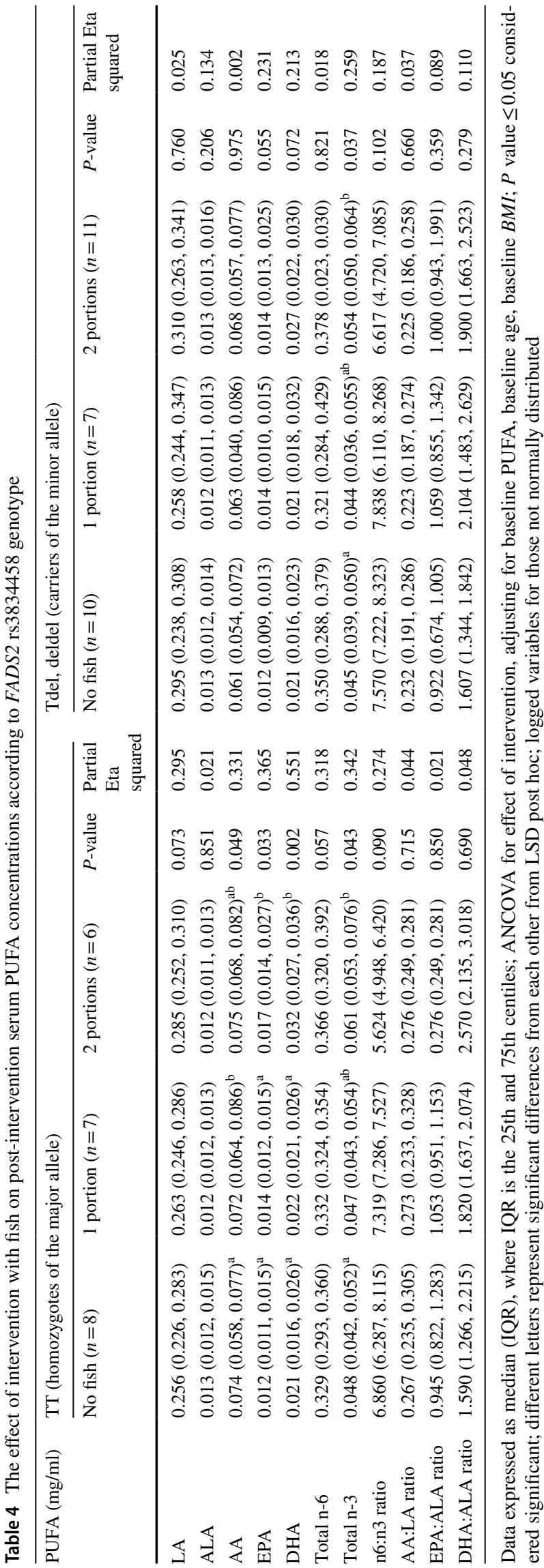

associations. Addressing the disparities amongst studies, we stratified intervention groups by FADS genotype, and thus controlled for the possibility of genetic confounding. We found that higher fish consumption resulted in increased serum n-3 PUFA concentrations. Given the importance of $n$-3 LCPUFA for the developing foetus during pregnancy, all pregnant women or those of childbearing age would benefit from consumption of long-chain n-3 PUFA, as reflected in current FDA guidelines for fish consumption [46].

The decrease in the $n-6: n-3$ ratio in this study is also of interest. This ratio is regarded as a biological marker with a higher $n-6: n-3$ ratio associated with increased risk of obesity [47], cardiovascular disease and inflammatory diseases [48]. Also, an increased maternal $n-6: n-3$ fatty acid ratio is associated with poorer child development [49]. The current study reports a significant decrease in $n-6: n-3$ ratio from 7.174 to 6.199 in those consuming two portions of fish per week. A ratio of 3 to 4 has been suggested to be potentially beneficial in preventing early neurodegeneration, cancer and cardiovascular disease [50]. We hypothesise that with greater consumption of fish the $n-6: n-3$ ratio may decrease further.

N-3 LCPUFA are associated with reduced inflammation [51] and a lowering of lipid profile, specifically triglycerides and low-density lipoprotein [52]. As fish is a rich source of LCPUFA, it has been suggested that an increase in fish consumption may be beneficial for inflammation [37], oxidative stress [38] and lipid profile [39]. However, despite increased $n-3$ LCPUFA and decreased $n-6: n-3$ ratios with 2 portions of fish consumed, the current study did not detect any significant differences in any of the biomarkers analysed between groups. The absence of an effect of fish consumption on these biomarkers may be owing to the inclusion of healthy participants and small sample size, as the study was not powered to determine an effect on biomarkers of inflammation, oxidative stress and lipids. Previous studies which have reported an association between fish and lipid profile and inflammatory markers have been observational studies using self-reported intake of fish [37, 38]. This approach may result in fish intake reported being over or underestimated compared to what was actually consumed. Some RCT trials have investigated the influence of fish on lipid profile; however, these were powered to investigate the effect of fish consumption on lipid markers [39], and may explain the discrepancies between these findings and those reported in the current analysis. In the current study, it may be the case that greater fish consumption may increase circulating concentrations of $n-3$, and thereby lower the ratio further to a ratio observed to be beneficial. This effect in turn may impact on biomarkers of inflammation, oxidative stress and lipids.

Results of our secondary analysis found there was no interaction between fish intervention and genotype. The lack of a fish intervention $\times$ genotype interaction may be owing to insufficient statistical power to test this. Response 
to fish intake, however, seemed to differ within the genotype groups. Participants in the two portions of fish group carrying the minor allele had significantly higher total $n$-3 PUFA concentrations post-intervention compared to those in the no fish group. Major allele homozygotes in the two portion per week group had higher serum total $n$-3 PUFA, EPA and DHA concentrations following the intervention period compared to those in the no fish group. Therefore, increased fish intake was found to increase serum $n$-3 PUFA concentrations in both genotype groups, albeit this increase was more pronounced in individuals homozygous for the major allele. These findings suggest that consuming 2 portions of fish per week may result in higher circulating PUFA concentrations depending on genotype; however, further research is needed to confirm this possibility. At post-intervention, carriers of the minor allele (Tdel, deldel) had significantly lower serum AA concentrations and a lower AA:LA ratio compared to major allele homozygotes. These data suggest that endogenous synthesis of AA is not as efficient in those who are minor allele carriers, a finding which has been shown previously in other cohorts [18, 23, 53]. No differences in post-intervention concentrations of the $n$-3 PUFA EPA or DHA were found for major allele homozygotes (TT) compared to carriers of the minor allele (Tdel, deldel) and is in agreement with previous studies which suggest DHA may be less influenced by variation in FADS genotype [18, 53]. When examined according to intervention group, those in the 2 portions of fish per week group and who were homozygous major allele carriers (TT) had a higher AA:LA and DHA:ALA ratio compared to minor allele carriers (Tdel, deldel). This finding is likely owing to more efficient endogenous synthesis of PUFA in major allele homozygotes, and in the case of DHA:ALA, the increased consumption of preformed DHA from 2 portions of fish per week has resulted in a higher ratio.

The presence of the minor allele has been linked to decreased synthesis of LCPUFA via the elongation pathway $[16,41]$. Some intervention studies have investigated the influence of PUFA supplementation on biological PUFA status according to FADS genotype, and reported that n-3 PUFA supplements increase DHA in minor allele carriers [54]. A previous intervention study investigated whether fish oil supplementation was associated with desaturase enzyme activity according to FADS genotype. Following supplementation there was an association between FADS genotype and increased D5D activity, suggesting n-3 PUFA supplementation may influence PUFA metabolism when genetic variation is present [55]. Dietary intake data collected at postintervention appointments show that there was no significant difference in dietary intake of PUFA between the genotype groups (Online Resource 3). Major allele homozygotes may synthesise LCPUFA more efficiently in the body from precursor molecules, which coupled with increased dietary intake could explain the increased biological concentrations at post-intervention. Individuals with certain genotypes, particularly minor allele carriers, may respond differently to fish consumption and thus further research is needed to investigate this possibility. The $n-6$ dietary intake of participants in the current study at post-intervention was $6.92 \mathrm{~g} / \mathrm{day}$ and $7.87 \mathrm{~g} /$ day for those with the Tdel and deldel genotypes, respectively. This intake is lower than previously reported $\mathrm{n}-6$ intakes of $8.6 \mathrm{~g} /$ day in the UK, and the European average of $11.9 \mathrm{~g} /$ day [56]. The lower intakes reported in the current study may in part be owing to the dietary assessment method used. A 24-h recall was used in the current study to record habitual food intake data; however, this is a limitation of the study as a food frequency questionnaire (FFQ) or weighed food dairy may have provided more robust dietary data. It is also possible that the dietary analysis software used did not contain detailed information on PUFA composition of foods.

Further limitations of the study include only screening for one particular genotype (rs3834458). Other FADS SNPs not in linkage disequilibrium with rs 3834458 could be considered. Genetic variation in the ELOVL genes which encode the elongase enzymes has been found to influence PUFA status [55] and therefore should be considered in future studies. PUFA analysis was completed in serum, which is considered to be reflective of dietary intake in approximately the last $24 \mathrm{~h}$ before sampling, however, measurement of PUFA in serum lipoproteins or erythrocyte membranes may have better reflected the intake over the intervention period. The sample size of the current study was not powered to investigate the influence of fish consumption on lipids or markers of inflammation and oxidative stress. Also, high trans-fatty acid (TFA) and saturated fatty acid status have been shown to mask some effects of PUFAs on oxidative stress, inflammation and lipids [57] but were not taken into account in the current analysis. This study also has many strengths. To our knowledge, this is the first intervention study investigating the influence of fish consumption on serum n-3 PUFA concentrations while allowing for a major confounding factor of genetic variation in FADS2. Also, compliance was high for the intervention study. GC-MS is regarded as the gold standard method for PUFA analysis which is a further strength of the study.

Various factors should be considered in future work such as the maximum amount of fish given, as higher consumption of fish may further increase PUFA status and warrants further investigation. The intervention period for the current study was 8 weeks as this was deemed suitable to see a change in PUFA status; however, it is possible that a longer intervention period may have allowed further changes in PUFA status to occur. In this study we chose to stratify by rs 3834458 to account for the effect of genotype during randomisation; however, future studies should consider screening participants for a particular genotype and 
intervening with these people to see how fish consumption influenced PUFA in that particular genotype.

\section{Conclusion}

This is the first intervention study to show that consumption of 2 portions of fish per week resulted in a significant increase in serum $n-3$ PUFA concentrations and a decrease in the $n-6: n-3$ ratio compared to consumption of no fish or one portion of fish in women of childbearing age while stratifying by FADS genotype. This study supports the current international public health guidelines to consume 2 portions of fish per week. Fish consumption was found to have no effect on markers of inflammation or oxidative stress in the current study; however, greater fish intake may be necessary to influence these biomarkers. This study, for the first time, stratified by FADS genotype during randomisation and, therefore, takes account of confounding from genotype so that the influence of fish consumption was not biased by any genetic effect. Future research in this area should investigate the effects of higher intakes of fish on PUFA status taking into consideration FADS genotype.

Author contributions MC, AY, MM, EMcS, JJS and EvW planned and designed the research and were involved in data interpretation. $\mathrm{MC}$ conducted the research including day-to-day running of the study and data collection. KB, DP and KW conducted genotyping analysis. $\mathrm{MC}$ and TS conducted all other laboratory analysis. $\mathrm{BH}$ and $\mathrm{DC}$ provided expert advice on GC-MS. MC and MM conducted the statistical analysis. MC drafted the manuscript, and all authors commented on previous versions of the manuscript. All authors read and approved the final manuscript.

Funding This research was supported by the Department for Employment and Learning (DEL), Northern Ireland, the US National Institutes of Health (NIH) (Grants R01-ES010219 and P30-ES01247), the Swedish Research Council FORMAS and the Karolinska Institutet, Sweden. The study sponsors had no role in the design, collection, analysis or interpretation of data, in the writing of the manuscript, or the decision to submit for publication.

\section{Compliance with ethical standard}

Conflict of interest The authors declare that they have no conflict of interest.

Ethics approval All participants provided written informed consent and the study was approved by Ulster University Research Ethics Committee (REC/16/0077), and all research conducted in accordance with the 1964 Declaration of Helsinki and its later amendments.

Consent to participate All participants gave their informed consent prior to their inclusion in the study.
Open Access This article is licensed under a Creative Commons Attribution 4.0 International License, which permits use, sharing, adaptation, distribution and reproduction in any medium or format, as long as you give appropriate credit to the original author(s) and the source, provide a link to the Creative Commons licence, and indicate if changes were made. The images or other third party material in this article are included in the article's Creative Commons licence, unless indicated otherwise in a credit line to the material. If material is not included in the article's Creative Commons licence and your intended use is not permitted by statutory regulation or exceeds the permitted use, you will need to obtain permission directly from the copyright holder. To view a copy of this licence, visit http://creativecommons.org/licenses/by/4.0/.

\section{References}

1. Calder PC (2015) Functional roles of fatty acids and their effects on human health. J Parenter Enter Nutr 39:18S-32S. https://doi. org/10.1177/0148607115595980

2. Innis SM (2007) Dietary (n-3) fatty acids and brain development. J Nutr 137:855-859. https://doi.org/10.1093/jn/137.4.855

3. Brenna JT, Diau GY (2007) The influence of dietary docosahexaenoic acid and arachidonic acid on central nervous system polyunsaturated fatty acid composition. Prostaglandins Leukot Essent Fat Acids 77:247-250. https://doi.org/10.1016/j.plefa .2007.10.016

4. Singh M (2005) Essential fatty acids, DHA, and the human brain. Indian J Pediatr 72:239-242

5. Makrides M (2011) The role of n-3 LCPUFA in pregnancy. OCL Ol Corps Gras Lipides 18:255-258. https://doi.org/10.1684/ ocl.2011.0408

6. Shahidi F, Ambigaipalan P (2018) Omega-3 polyunsaturated fatty acids and their health benefits. Annu Rev Food Sci Technol 9:345-381. https://doi.org/10.1146/annurev-food-111317-09585 0

7. Judge MP, Harel O, Lammi-Keefe CJ (2007) Maternal consumption of a docosahexaenoic acid-containing functional food during pregnancy: Benefit for infant performance on problemsolving but not on recognition memory tasks at age 9 mo. Am J Clin Nutr 85:1572-1577. https://doi.org/10.1093/ajcn/85.6.1572

8. Dunstan JA, Simmer K, Dixon G, Prescott SL (2008) Cognitive assessment of children at age $21 / 2$ years after maternal fish oil supplementation in pregnancy: A randomised controlled trial. Arch Dis Child Fetal Neonatal Ed. https://doi.org/10.1136/ adc. 2006.099085

9. Bonham MP, Duffy EM, Wallace JMW et al (2008) Habitual fish consumption does not prevent a decrease in LCPUFA status in pregnant women (the Seychelles Child Development Nutrition Study). Prostaglandins Leukot Essent Fat Acids 78:343-350. https://doi.org/10.1016/j.plefa.2008.04.005

10. Strain JJ, Davidson PW, Bonham MP et al (2008) Associations of maternal long-chain polyunsaturated fatty acids, methyl mercury, and infant development in the Seychelles Child Development Nutrition Study. Neurotoxicology 29:776-782. https://doi. org/10.1016/j.neuro.2008.06.002

11. Strain JJ, Davidson PW, Thurston SW et al (2012) Maternal PUFA status but not prenatal methylmercury exposure is associated with children's language functions at age five years in the seychelles. J Nutr 142:1943-1949. https://doi.org/10.3945/jn.112.163493

12. Strain JJ, Yeates AJ, Van Wijngaarden E et al (2015) Prenatal exposure to methyl mercury from fish consumption and polyunsaturated fatty acids: Associations with child development at 20 mo of age in an observational study in the Republic of Seychelles. Am J Clin Nutr 101:530-537. https://doi.org/10.3945/ ajcn.114.100503 
13. Calder PC (2017) Omega-3 fatty acids and inflammatory processes: From molecules to man. Biochem Soc Trans 45:11051115. https://doi.org/10.1042/BST20160474

14. Fan C, Zirpoli H, Qi K (2013) N-3 fatty acids modulate adipose tissue inflammation and oxidative stress. Curr Opin Clin Nutr Metab Care 16:124-132. https://doi.org/10.1097/MCO.0b013 e32835c02c8

15. Leslie MA, Cohen DJA, Liddle DM et al (2015) A review of the effect of omega-3 polyunsaturated fatty acids on blood triacylglycerol levels in normolipidemic and borderline hyperlipidemic individuals. Lipids Health Dis. https://doi.org/10.1186/s1294 4-015-0049-7

16. Glaser C, Heinrich J, Koletzko B (2010) Role of FADS1 and FADS2 polymorphisms in polyunsaturated fatty acid metabolism. Metabolism 59:993-999. https://doi.org/10.1016/j.metab ol.2009.10.022

17. Mathias RA, Pani V, Chilton FH (2014) Genetic Variants in the FADS Gene: Implications for Dietary Recommendations for Fatty Acid Intake. Curr Nutr Rep 3:139-148. https://doi. org/10.1007/s13668-014-0079-1

18. Yeates AJ, Love TM, Engström K et al (2015) Genetic variation in FADS genes is associated with maternal long-chain PUFA status but not with cognitive development of infants in a high fish-eating observational study. Prostaglandins Leukot Essent Fat Acids 102-103:13-20. https://doi.org/10.1016/j.plefa 2015.08.004

19. Tanaka T, Shen J, Abecasis GR et al (2009) Genome-wide association study of plasma polyunsaturated fatty acids in the InCHIANTI study. PLoS Genet 5:1-8. https://doi.org/10.1371/journ al.pgen. 1000338

20. Malerba G, Schaeffer L, Xumerle L et al (2008) SNPs of the FADS gene cluster are associated with polyunsaturated fatty acids in a cohort of patients with cardiovascular disease. Lipids 43:289-299. https://doi.org/10.1007/s11745-008-3158-5

21. Li P, Zhao J, Kothapalli KSD et al (2018) A regulatory insertiondeletion polymorphism in the FADS gene cluster influences PUFA and lipid profiles among Chinese adults: A population-based study. Am J Clin Nutr 107:867-875. https://doi.org/10.1093/ajen/ nqy063

22. Moltó-Puigmartí C, Plat J, Mensink RP et al (2010) FADS1 FADS2 gene variants modify the association between fish intake and the docosahexaenoic acid proportions in human milk. Am J Clin Nutr 91:1368-1376. https://doi.org/10.3945/ajen.2009.28789

23. Schaeffer L, Gohlke H, Müller M et al (2006) Common genetic variants of the FADS1 FADS2 gene cluster and their reconstructed haplotypes are associated with the fatty acid composition in phospholipids. Hum Mol Genet 15:1745-1756. https://doi. org $/ 10.1093 / \mathrm{hmg} / \mathrm{dd} 1117$

24. Ensembl (2018) No Title. www.ensembl.org

25. Murff HJ, Edwards TL (2014) Endogenous production of longchain polyunsaturated fatty acids and metabolic disease risk. Curr Cardiovasc Risk Rep 8:418. https://doi.org/10.1007/s1217 0-014-0418-1

26. Bates B, Cox L, Nicholson S, et al (2016) National diet and nutrition survey: results from years 5 and 6.6

27. Amiano P, Dorronsoro M, De Renobales M et al (2001) Verylong-chain $\omega$-3 fatty acids as markers for habitual fish intake in a population consuming mainly lean fish: The EPIC cohort of Gipuzkoa. Eur J Clin Nutr 55:827-832. https://doi.org/10.1038/ sj.ejen. 1601242

28. Brantsaeter AL, Haugen M, Thomassen Y et al (2010) Exploration of biomarkers for total fish intake in pregnant Norwegian women. Public Health Nutr 13:54-62. https://doi.org/10.1017/S136898000 9005904

29. Parra M-S, Schnaas L, Meydani M et al (2002) Erythrocyte cell membrane phospholipid levels compared against reported dietary intakes of polyunsaturated fatty acids in pregnant Mexican women. Public Health Nutr 5:931-937. https://doi.org/10.1079/ phn2002381

30. Bonaa KH, Bjerve KS, Nordoy A (1992) Habitual fish consumption, plasma phospholipid fatty acids, and serum lipids: The Tromso study. Am J Clin Nutr 55:1126-1134. https://doi. org/10.1093/ajen/55.6.1126

31. Fisk HL, Irvine M, Miles EA et al (2018) Association of oily fish intake, sex, age, BMI and APOE genotype with plasma longchain n-3 fatty acid composition. Br J Nutr 120:23-32. https://doi. org/10.1017/S000711451800106X

32. Philibert A, Vanier C, Abdelouahab N et al (2006) Fish intake and serum fatty acid profiles from freshwater fish. Am J Clin Nutr 84:1299-1307. https://doi.org/10.1093/ajen/84.6.1299

33. Garcia-Rodriguez CE, Olza J, Mesa MD et al (2017) Fatty acid status and antioxidant defense system in mothers and their newborns after salmon intake during late pregnancy. Nutrition 33:157-162. https://doi.org/10.1016/j.nut.2016.05.015

34. Miles EA, Noakes PS, Kremmyda LS et al (2011) The Salmon in Pregnancy Study: Study design, subject characteristics, maternal fish and marine n-3 fatty acid intake, and marine n-3 fatty acid status in maternal and umbilical cord blood. Am J Clin Nutr 94:1986-1992. https://doi.org/10.3945/ajen.110.001636

35. De Groot RHM, Emmett R, Meyer BJ (2019) Non-dietary factors associated with n-3 long-chain PUFA levels in humans - A systematic literature review. Br J Nutr 121:793-808. https://doi. org/10.1017/S0007114519000138

36. Welch AA, Shakya-Shrestha S, Lentjes MAH et al (2010) Dietary intake and status of n-3 polyunsaturated fatty acids in a population of fish-eating and non-fish-eating meat-eaters, vegetarians, and vegans and the precursor-product ratio of $\alpha$-linolenic acid to long-chain n-3 polyunsaturated fatty acids: Results. Am J Clin Nutr 92:1040-1051. https://doi.org/10.3945/ajen.2010.29457

37. Zampelas A, Panagiotakos DB, Pitsavos C et al (2005) Fish consumption among healthy adults is associated with decreased levels of inflammatory markers related to cardiovascular disease: The ATTICA study. J Am Coll Cardiol 46:120-124. https://doi. org/10.1016/j.jacc.2005.03.048

38. Hansson P, Barregård L, Halltorp $M$ et al (2015) Habitual high intake of fatty fish is related to lower levels of F2-isoprostane in healthy women. Nutrition 31:847-852. https://doi.org/10.1016/j. nut.2014.12.015

39. Zibaeenezhad MJ, Ghavipisheh M, Attar A, Aslani A (2017) Comparison of the effect of omega-3 supplements and fresh fish on lipid profile: A randomized, open-labeled trial. Nutr Diabetes 7:1-8. https://doi.org/10.1038/s41387-017-0007-8

40. Harsløf LB, Larsen LH, Ritz C et al (2013) FADS genotype and diet are important determinants of DHA status: a cross-sectional study in Danish infants. Am J Clin Nutr 97:1403-1410. https:// doi.org/10.3945/ajen.113.058685

41. Al-Hilal M, AlSaleh A, Maniou Z et al (2013) Genetic variation at the FADS1-FADS2 gene locus influences delta-5 desaturase activity and LC-PUFA proportions after fish oil supplement. J Lipid Res 54:542-551. https://doi.org/10.1194/jlr.P032276

42. Folch J, Lees M, Stanley GHS (1957) A simple method for the isolation and purification of total lipides from animal tissues. $\mathrm{J}$ Biol Chem 226:497-509

43. Morrison WR, Smith LM (1964) Preparation of fatty acid methyl esters and dimethylacetals from lipids. J Lipid Res 5:600-608

44. Moher D, Hopewell S, Schulz KF et al (2010) CONSORT 2010 explanation and elaboration: updated guidelines for reporting parallel group randomised trials. BMJ. https://doi.org/10.1136/bmj. c869

45. SACN (2004) Advice on fish consumption: benefits \& risks 2004 . TSO, United Kingdom 
46. Food \& Drug Administration (2017) Advice about Eating Fish: For Women Who Are of Might Become Pregnant, Breastfeeding Mothers, and Young Children. https://www.fda.gov/food/consu mers/advice-about-eating-fish. Accessed 28 Nov 2018

47. Simopoulos AP (2016) An increase in the Omega-6/Omega-3 fatty acid ratio increases the risk for obesity. Nutrients 8:1-17. https:// doi.org/10.3390/nu8030128

48. Simopoulos AP (2011) Evolutionary aspects of diet: The omega-6/ omega-3 ratio and the brain. Mol Neurobiol 44:203-215. https:// doi.org/10.1007/s12035-010-8162-0

49. Bernard JY, De Agostini M, Forhan A et al (2013) The Dietary n6:n3 Fatty Acid Ratio during Pregnancy Is Inversely Associated with Child Neurodevelopment in the EDEN Mother-Child Cohort. J Nutr 143:1481-1488. https://doi.org/10.3945/jn.113.178640

50. Simopoulos AP (2010) The omega-6/omega-3 fatty acid ratio: Health implications. OCL - Ol Corps Gras Lipides 17:267-275. https://doi.org/10.1684/ocl.2010.0325

51. Calder PC (2012) Mechanisms of Action of (n-3) Fatty Acids. J Nutr 142:592S-599S. https://doi.org/10.3945/jn.111.155259

52. Adkins Y, Kelley DS (2010) Mechanisms underlying the cardioprotective effects of omega-3 polyunsaturated fatty acids. J Nutr Biochem 21:781-792. https://doi.org/10.1016/j.jnutb io. 2009.12.004

53. Koletzko B, Lattka E, Zeilinger S et al (2011) Genetic variants of the fatty acid desaturase gene cluster predict amounts of red blood cell docosahexaenoic and other polyunsaturated fatty acids in pregnant women: Findings from the Avon Longitudinal Study of Parents and Children. Am J Clin Nutr 93:211-219. https://doi. org/10.3945/ajen.110.006189

54. Meldrum SJ, Li Y, Zhang G et al (2018) Can polymorphisms in the fatty acid desaturase (FADS) gene cluster alter the effects of fish oil supplementation on plasma and erythrocyte fatty acid profiles? An exploratory study. Eur J Nutr 57:2583-2594. https:// doi.org/10.1007/s00394-017-1529-5

55. Cormier H, Rudkowska I, Lemieux S et al (2014) Effects of FADS and ELOVL polymorphisms on indexes of desaturase and elongase activities: Results from a pre-post fish oil supplementation. Genes Nutr. https://doi.org/10.1007/s12263-014-0437-z

56. Rippin HL, Hutchinson J, Jewell J et al (2017) Adult nutrient intakes from current national dietary surveys of european populations. Nutrients 9:1-47. https://doi.org/10.3390/nu9121288

57. Menaa F, Menaa A, Menaa B, Tréton J (2013) Trans-fatty acids, dangerous bonds for health? A background review paper of their use, consumption, health implications and regulation in France. Eur J Nutr 52:1289-1302. https://doi.org/10.1007/s0039 4-012-0484-4 